Supporting Information

\title{
Unexpected Reactions Between Ziegler-Natta Catalyst Components and Structural Characterization of Resulting Intermediates
}

Piotr Sobota,* Józef Utko, Tadeusz Lis, Łukasz John, Rafał Petrus, and Anna Drąg-Jarząbek

Faculty of Chemistry, University of Wrocław, 14 F. Joliot-Curie, 50-383 Wrocław, Poland. 
Table S1. Crystal and data collection parameters for compounds 2-5.

\begin{tabular}{|c|c|c|c|c|c|c|}
\hline & 2 & 3 & $\mathbf{3 \cdot 3 a} \cdot 0.5 \mathrm{EtOH}$ & 4 & $4 a$ & $\mathbf{5} \cdot 2 \mathrm{EtOH}$ \\
\hline Chemical formula & $\mathrm{C}_{12} \mathrm{H}_{28} \mathrm{Cl}_{2} \mathrm{MgO}_{4}$ & $\mathrm{C}_{30} \mathrm{H}_{81} \mathrm{Cl}_{3} \mathrm{Mg}_{4} \mathrm{O}_{19} \mathrm{Ti}_{3}$ & $\mathrm{C}_{33} \mathrm{H}_{88} \mathrm{Cl}_{3} \mathrm{Mg}_{4} \mathrm{O}_{19.5} \mathrm{Ti}_{3}$ & $\mathrm{C}_{32} \mathrm{H}_{84} \mathrm{Mg}_{2} \mathrm{O}_{16} \mathrm{Ti}_{2}$ & $\mathrm{C}_{32} \mathrm{H}_{84} \mathrm{Mg}_{2} \mathrm{O}_{16} \mathrm{Ti}_{2}$ & $\mathrm{C}_{44} \mathrm{H}_{118} \mathrm{Cl}_{2} \mathrm{Mg}_{6} \mathrm{O}_{28} \mathrm{Ti}_{4}$ \\
\hline Formula Mass & 331.55 & 1093.23 & 1144.32 & 869.35 & 869.41 & 1503.74 \\
\hline Crystal system & Monoclinic & Monoclinic & Monoclinic & Triclinic & Triclinic & Triclinic \\
\hline Space group & $P 2_{1} / n$ & $P 2_{1} / n$ & $C 2 / c$ & $P \overline{1}$ & $P \overline{1}$ & $P \overline{1}$ \\
\hline$a / \AA$ & $6.192(3)$ & $12.248(3)$ & $20.172(5)$ & $9.270(3)$ & $10.113(3)$ & $12.107(2)$ \\
\hline$b / \AA$ & $16.614(4)$ & $27.353(6)$ & $19.331(5)$ & $11.398(3)$ & 11.781(4) & $12.314(3)$ \\
\hline$c / \AA$ & $16.832(5)$ & $15.776(4)$ & $29.658(6)$ & $11.664(4)$ & $11.905(4)$ & $14.175(3)$ \\
\hline$\alpha /^{\circ}$ & & & & $71.80(5)$ & $68.89(5)$ & $111.63(3)$ \\
\hline$\beta /{ }^{\circ}$ & $96.50(3)$ & $95.97(2)$ & $101.69(2)$ & $78.16(5)$ & $86.47(5)$ & 109.24(3) \\
\hline$\gamma /{ }^{\circ}$ & & & & $89.30(5)$ & $70.01(5)$ & $94.39(3)$ \\
\hline Unit cell volume $/ \AA^{3}$ & $1720.4(11)$ & $5257(2)$ & $11325(5)$ & $1144.1(7)$ & $1240.4(9)$ & $1806.9(10)$ \\
\hline Temperature/K & $100(2)$ & $120(2)$ & $80(2)$ & $100(2)$ & $280(2)$ & $100(2)$ \\
\hline$Z$ & 4 & 4 & 8 & 1 & 1 & 1 \\
\hline Radiation type & $\mathrm{MoK} \alpha$ & $\mathrm{MoK} \alpha$ & $\mathrm{MoK} \alpha$ & $\mathrm{MoK} \alpha$ & $\mathrm{MoK} \alpha$ & $\mathrm{MoK} \alpha$ \\
\hline Absorption coefficient, $\mu / \mathrm{mm}^{-1}$ & 0.42 & 0.707 & 0.66 & 0.436 & 0.402 & 0.624 \\
\hline No. of reflections measured & 13649 & 38539 & 30478 & 6696 & 8801 & 13756 \\
\hline No. of independent reflections & 5032 & 12869 & 13070 & 4726 & 5465 & 7128 \\
\hline No. of obsevred reflections $(I>2 \sigma(I))$ & 4369 & 8469 & 9264 & 2648 & 2354 & 3115 \\
\hline$R_{\text {int }}$ & 0.0219 & 0.0524 & 0.0306 & 0.048 & 0.065 & 0.0799 \\
\hline Final $R_{l}$ values $(I>2 \sigma(I))$ & 0.0345 & 0.0447 & 0.0593 & 0.0773 & 0.084 & 0.0846 \\
\hline Final $w R\left(F^{2}\right)$ values $(I>2 \sigma(I))$ & 0.0773 & 0.1075 & 0.1462 & 0.175 & 0.2056 & 0.1786 \\
\hline Final $R_{l}$ values (all data) & 0.0432 & 0.0746 & 0.0901 & 0.1452 & 0.1678 & 0.1959 \\
\hline Final $w R\left(F^{2}\right)$ values (all data) & 0.0816 & 0.1153 & 0.1652 & 0.2164 & 0.2845 & 0.2356 \\
\hline Goodness of fit on $F^{2}$ & 1.062 & 0.919 & 1.031 & 1.022 & 0.982 & 0.995 \\
\hline$\Delta \rho \max / \mathrm{e} \AA^{-3}$ & 0.77 & 0.43 & 0.59 & 0.76 & 0.31 & 0.44 \\
\hline$\Delta \rho \min / \mathrm{e}^{-3}$ & -0.31 & -0.43 & -0.42 & -0.54 & -0.34 & -0.40 \\
\hline
\end{tabular}




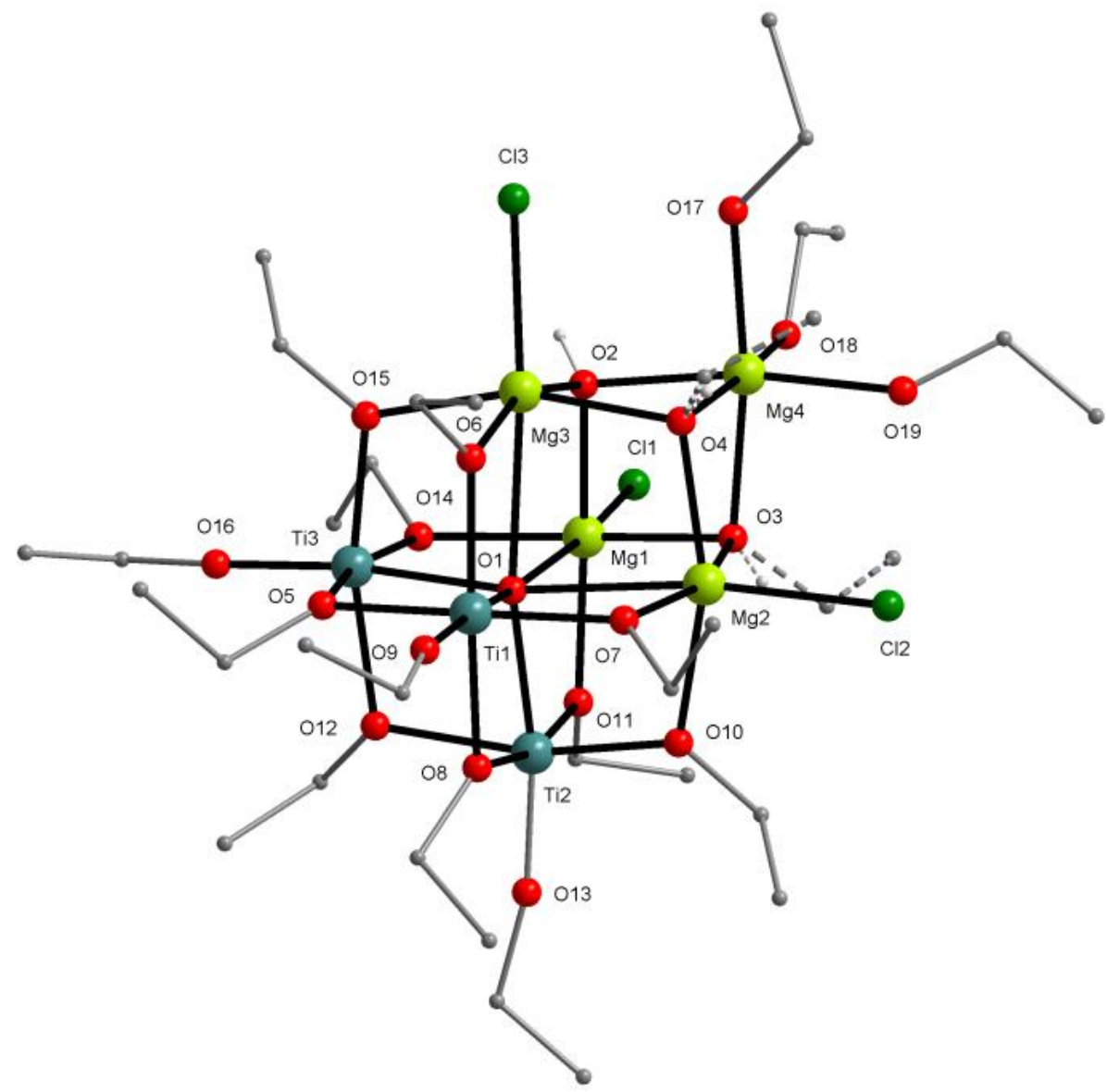

Figure S1. Molecular structure of $\left[\mathrm{Mg}_{4} \mathrm{Ti}_{3}\left(\mu_{6}-\mathrm{O}\right)\left(\mu_{3}-\mathrm{OH}\right)_{3}(\mu-\mathrm{OEt})_{9}(\mathrm{OEt})_{3}(\mathrm{EtOH})_{3} \mathrm{Cl}_{3}\right]$ $\cdot\left[\mathrm{Mg}_{4} \mathrm{Ti}_{3}\left(\mu_{6}-\mathrm{O}\right)\left(\mu_{3}-\mathrm{OH}\right)\left(\mu_{3}-\mathrm{OEt}\right)_{2}(\mu-\mathrm{OEt})_{9}(\mathrm{OEt})_{3}(\mathrm{EtOH})_{3} \mathrm{Cl}_{3}\right](\mathbf{3} \cdot \mathbf{3 a})$. Solvent molecules, and hydrogen atoms are omitted for clarity. 
Table S2. Selected geometric parameters for compounds 2-5 $\left(\AA,^{\circ}\right)$.

\begin{tabular}{|c|c|c|c|}
\hline \multicolumn{4}{|c|}{2} \\
\hline $\mathrm{Mg} 1-\mathrm{O} 1$ & $2.1235(11)$ & $\mathrm{Mg} 1-\mathrm{O} 4$ & $2.0779(11)$ \\
\hline $\mathrm{Mg} 1-\mathrm{O} 2$ & $2.1307(11)$ & $\mathrm{Mg} 1-\mathrm{Cl} 1$ & $2.4566(9)$ \\
\hline $\mathrm{Mg} 1-\mathrm{O} 3$ & $2.0616(11)$ & $\mathrm{Mg} 1-\mathrm{Cl} 2$ & $2.4670(9)$ \\
\hline $\mathrm{O} 1-\mathrm{Mg} 1-\mathrm{O} 2$ & $178.30(4)$ & $\mathrm{O} 2-\mathrm{Mg} 1-\mathrm{O} 4$ & $90.30(5)$ \\
\hline $\mathrm{O} 1-\mathrm{Mg} 1-\mathrm{O} 3$ & $91.91(5)$ & $\mathrm{O} 3-\mathrm{Mg} 1-\mathrm{O} 4$ & $177.52(4)$ \\
\hline $\mathrm{O} 1-\mathrm{Mg} 1-\mathrm{O} 4$ & $90.12(5)$ & $\mathrm{O} 3-\mathrm{Mg} 1-\mathrm{Cl}$ & $93.46(4)$ \\
\hline $\mathrm{O} 1-\mathrm{Mg} 1-\mathrm{Cl} 1$ & $89.16(4)$ & $\mathrm{O} 3-\mathrm{Mg} 1-\mathrm{Cl} 2$ & $87.04(4)$ \\
\hline $\mathrm{O} 1-\mathrm{Mg} 1-\mathrm{Cl} 2$ & $90.63(4)$ & $\mathrm{O} 4-\mathrm{Mg} 1-\mathrm{Cl}$ & $87.99(4)$ \\
\hline $\mathrm{O} 2-\mathrm{Mg} 1-\mathrm{O} 3$ & $87.72(5)$ & $\mathrm{O} 4-\mathrm{Mg} 1-\mathrm{Cl} 2$ & $91.52(4)$ \\
\hline $\mathrm{O} 2-\mathrm{Mg} 1-\mathrm{Cl} 1$ & $89.21(4)$ & $\mathrm{Cl} 1-\mathrm{Mg} 1-\mathrm{Cl} 2$ & $179.47(2)$ \\
\hline $\mathrm{O} 2-\mathrm{Mg} 1-\mathrm{Cl} 2$ & $91.0(4)$ & & \\
\hline \multicolumn{4}{|l|}{ 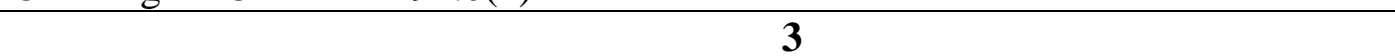 } \\
\hline $\mathrm{Ti1}-\mathrm{O} 1$ & $2.1161(16)$ & $\mathrm{Mg} 1-\mathrm{O} 11$ & $2.0503(19)$ \\
\hline $\mathrm{Ti1}-\mathrm{O} 5$ & $2.0043(17)$ & $\mathrm{Mg} 1-\mathrm{O} 14$ & $2.0507(19)$ \\
\hline Ti1-O6 & $1.9239(17)$ & $\mathrm{Mg} 1-\mathrm{Cl} 1$ & $2.4206(12)$ \\
\hline Ti1-O7 & $1.9357(17)$ & $\mathrm{Mg} 2-\mathrm{O} 1$ & $2.4829(17)$ \\
\hline $\mathrm{Ti1}-\mathrm{O} 8$ & $2.0273(17)$ & $\mathrm{Mg} 2-\mathrm{O} 3$ & $2.0433(18)$ \\
\hline Ti1-O9 & $1.7675(17)$ & $\mathrm{Mg} 2-\mathrm{O} 4$ & $2.0540(18)$ \\
\hline $\mathrm{Ti} 2-\mathrm{O} 1$ & $2.0855(16)$ & $\mathrm{Mg} 2-\mathrm{O} 7$ & $2.0482(18)$ \\
\hline $\mathrm{Ti} 2-\mathrm{O} 8$ & $2.0279(17)$ & $\mathrm{Mg} 2-\mathrm{O} 10$ & $2.0583(18)$ \\
\hline $\mathrm{Ti} 2-\mathrm{O} 10$ & $1.9443(17)$ & $\mathrm{Mg} 2-\mathrm{Cl} 2$ & $2.4159(11)$ \\
\hline $\mathrm{Ti} 2-\mathrm{O} 11$ & $1.9442(17)$ & $\mathrm{Mg} 3-\mathrm{O} 1$ & $2.5396(18)$ \\
\hline $\mathrm{Ti} 2-\mathrm{O} 12$ & $1.9999(17)$ & $\mathrm{Mg} 3-\mathrm{O} 2$ & $2.0592(18)$ \\
\hline $\mathrm{Ti} 2-\mathrm{O} 13$ & $1.7751(18)$ & $\mathrm{Mg} 3-\mathrm{O} 4$ & $2.0362(18)$ \\
\hline $\mathrm{Ti} 3-\mathrm{O} 1$ & $2.1110(16)$ & $\mathrm{Mg} 3-\mathrm{O} 6$ & $2.0470(18)$ \\
\hline Ti3-O5 & 2.0293(17) & $\mathrm{Mg} 3-\mathrm{O} 15$ & $2.0475(18)$ \\
\hline $\mathrm{Ti3}-\mathrm{O} 12$ & 2.0291(17) & $\mathrm{Mg} 3-\mathrm{Cl} 3$ & $2.4147(11)$ \\
\hline $\mathrm{Ti} 3-\mathrm{O} 14$ & $1.9399(17)$ & $\mathrm{Mg} 4-\mathrm{O} 2$ & $2.0688(18)$ \\
\hline $\mathrm{Ti3}-\mathrm{O} 15$ & $1.9375(17)$ & $\mathrm{Mg} 4-\mathrm{O} 3$ & $2.0580(19)$ \\
\hline $\mathrm{Ti3}-\mathrm{O} 16$ & $1.7580(17)$ & $\mathrm{Mg} 4-\mathrm{O} 4$ & $2.0629(19)$ \\
\hline $\mathrm{Mg} 1-\mathrm{O} 1$ & $2.5246(18)$ & $\mathrm{Mg} 4-\mathrm{O} 17$ & $2.062(2)$ \\
\hline $\mathrm{Mg} 1-\mathrm{O} 2$ & $2.0470(19)$ & $\mathrm{Mg} 4-\mathrm{O} 18$ & $2.081(2)$ \\
\hline $\mathrm{Mg} 1-\mathrm{O} 3$ & $2.0565(18)$ & $\mathrm{Mg} 4-\mathrm{O} 19$ & $2.070(2)$ \\
\hline $\mathrm{O} 1-\mathrm{Ti} 1-\mathrm{O} 5$ & $78.13(6)$ & $\mathrm{O} 2-\mathrm{Mg} 1-\mathrm{Cl1}$ & $99.14(6)$ \\
\hline O1-Ti1-O6 & $87.37(7)$ & $\mathrm{O} 3-\mathrm{Mg} 1-\mathrm{O} 11$ & $91.35(7)$ \\
\hline $\mathrm{O} 1-\mathrm{Ti} 1-\mathrm{O} 7$ & $87.04(6)$ & $\mathrm{O} 3-\mathrm{Mg} 1-\mathrm{O} 14$ & $158.01(8)$ \\
\hline $\mathrm{O} 1-\mathrm{Ti} 1-\mathrm{O} 8$ & $77.67(6)$ & $\mathrm{O} 3-\mathrm{Mg} 1-\mathrm{Cl} 1$ & $97.73(6)$ \\
\hline O1-Ti1-O9 & $171.73(7)$ & $\mathrm{O} 11-\mathrm{Mg} 1-\mathrm{O} 14$ & $88.09(7)$ \\
\hline $\mathrm{O} 5-\mathrm{Ti1}-\mathrm{O} 6$ & $89.92(7)$ & $\mathrm{O} 11-\mathrm{Mg} 1-\mathrm{Cl} 1$ & $101.79(6)$ \\
\hline $\mathrm{O} 5-\mathrm{Ti} 1-\mathrm{O} 7$ & $165.14(7)$ & $\mathrm{O} 14-\mathrm{Mg} 1-\mathrm{Cl}$ & $103.91(6)$ \\
\hline O5- & $87.80(7)$ & $\mathrm{O} 1-\mathrm{Mg} 2-\mathrm{O} 3$ & $84.78(6)$ \\
\hline O5- & $96.11(7)$ & $\mathrm{O} 1-\mathrm{Mg} 2-\mathrm{O} 4$ & $84.91(6)$ \\
\hline O6-Ti1-O7 & $90.38(7)$ & $\mathrm{O} 1-\mathrm{Mg} 2-\mathrm{O} 7$ & $75.43(6)$ \\
\hline O6-Ti1-O8 & $165.02(7)$ & $\mathrm{O} 1-\mathrm{Mg} 2-\mathrm{O} 10$ & $75.98(6)$ \\
\hline O6-Ti1-O9 & $98.64(8)$ & $\mathrm{O} 1-\mathrm{Mg} 2-\mathrm{Cl} 2$ & $174.04(5)$ \\
\hline O7-Ti1-O8 & $88.09(7)$ & $\mathrm{O} 3-\mathrm{Mg} 2-\mathrm{O} 4$ & $79.71(7)$ \\
\hline O7-Ti1-O9 & $98.53(7)$ & $\mathrm{O} 3-\mathrm{Mg} 2-\mathrm{O} 7$ & $159.41(7)$ \\
\hline
\end{tabular}




\begin{tabular}{|c|c|c|c|}
\hline O8-Ti1-O9 & $96.32(7)$ & $\mathrm{O} 3-\mathrm{Mg} 2-\mathrm{O} 10$ & $92.08(7)$ \\
\hline $\mathrm{O} 1-\mathrm{Ti} 2-\mathrm{O} 8$ & $78.37(7)$ & $\mathrm{O} 3-\mathrm{Mg} 2-\mathrm{Cl} 2$ & $101.04(6)$ \\
\hline $\mathrm{O} 1-\mathrm{Ti} 2-\mathrm{O} 10$ & $88.56(6)$ & $\mathrm{O} 4-\mathrm{Mg} 2-\mathrm{O} 7$ & $92.71(7)$ \\
\hline $\mathrm{O} 1-\mathrm{Ti} 2-\mathrm{O} 11$ & $87.42(7)$ & $\mathrm{O} 4-\mathrm{Mg} 2-\mathrm{O} 10$ & $159.85(8)$ \\
\hline $\mathrm{O} 1-\mathrm{Ti} 2-\mathrm{O} 12$ & $78.45(6)$ & $\mathrm{O} 4-\mathrm{Mg} 2-\mathrm{Cl} 2$ & $97.28(6)$ \\
\hline $\mathrm{O} 1-\mathrm{Ti} 2-\mathrm{O} 13$ & $172.19(8)$ & $\mathrm{O} 7-\mathrm{Mg} 2-\mathrm{O} 10$ & $88.66(7)$ \\
\hline $\mathrm{O} 8-\mathrm{Ti} 2-\mathrm{O} 10$ & $90.11(7)$ & $\mathrm{O} 7-\mathrm{Mg} 2-\mathrm{Cl} 2$ & $98.88(6)$ \\
\hline $\mathrm{O} 8$ - Ti2-O11 & $165.78(7)$ & $\mathrm{O} 10-\mathrm{Mg} 2-\mathrm{Cl} 2$ & $102.37(6)$ \\
\hline $\mathrm{O} 8-\mathrm{Ti} 2-\mathrm{O} 12$ & $88.12(7)$ & $\mathrm{O} 1-\mathrm{Mg} 3-\mathrm{O} 2$ & $84.28(6)$ \\
\hline $\mathrm{O} 8-\mathrm{Ti} 2-\mathrm{O} 13$ & $95.96(8)$ & $\mathrm{O} 1-\mathrm{Mg} 3-\mathrm{O} 4$ & $83.80(6)$ \\
\hline $\mathrm{O} 10-\mathrm{Ti2}-\mathrm{O} 11$ & $88.99(7)$ & $\mathrm{O} 1-\mathrm{Mg} 3-\mathrm{O} 6$ & $74.18(6)$ \\
\hline $\mathrm{O} 10-\mathrm{Ti2}-\mathrm{O} 12$ & 166.99(7) & $\mathrm{O} 1-\mathrm{Mg} 3-\mathrm{O} 15$ & $74.51(6)$ \\
\hline $\mathrm{O} 10-\mathrm{Ti} 2-\mathrm{O} 13$ & $96.89(8)$ & $\mathrm{O} 1-\mathrm{Mg} 3-\mathrm{Cl} 3$ & $175.55(5)$ \\
\hline $\mathrm{O} 11-\mathrm{Ti2}-\mathrm{O} 12$ & $89.57(7)$ & $\mathrm{O} 2-\mathrm{Mg} 3-\mathrm{O} 4$ & $80.25(7)$ \\
\hline $\mathrm{O} 11-\mathrm{Ti} 2-\mathrm{O} 13$ & $98.23(8)$ & $\mathrm{O} 2-\mathrm{Mg} 3-\mathrm{O} 6$ & $157.80(7)$ \\
\hline $\mathrm{O} 12-\mathrm{Ti2}-\mathrm{O} 13$ & $96.12(8)$ & $\mathrm{O} 2-\mathrm{Mg} 3-\mathrm{O} 15$ & $91.83(7)$ \\
\hline $\mathrm{O} 1-\mathrm{Ti} 3-\mathrm{O} 5$ & $77.71(6)$ & $\mathrm{O} 2-\mathrm{Mg} 3-\mathrm{Cl} 3$ & $98.41(6)$ \\
\hline $\mathrm{O} 1-\mathrm{Ti} 3-\mathrm{O} 12$ & $77.23(6)$ & $\mathrm{O} 4-\mathrm{Mg} 3-\mathrm{O} 6$ & $91.92(7)$ \\
\hline $\mathrm{O} 1-\mathrm{Ti} 3-\mathrm{O} 14$ & $88.06(6)$ & $\mathrm{O} 4-\mathrm{Mg} 3-\mathrm{O} 15$ & $157.57(8)$ \\
\hline $\mathrm{O} 1-\mathrm{Ti} 3-\mathrm{O} 15$ & $87.58(6)$ & $\mathrm{O} 4-\mathrm{Mg} 3-\mathrm{Cl} 3$ & $100.12(6)$ \\
\hline $\mathrm{O} 1-\mathrm{Ti} 3-\mathrm{O} 16$ & $169.53(8)$ & $\mathrm{O} 6-\mathrm{Mg} 3-\mathrm{O} 15$ & $87.64(7)$ \\
\hline $\mathrm{O} 5-\mathrm{Ti} 3-\mathrm{O} 12$ & $87.32(7)$ & $\mathrm{O} 6-\mathrm{Mg} 3-\mathrm{Cl} 3$ & $103.42(6)$ \\
\hline $\mathrm{O} 5-\mathrm{Ti} 3-\mathrm{O} 14$ & $165.78(7)$ & $\mathrm{O} 15-\mathrm{Mg} 3-\mathrm{Cl} 3$ & $101.79(6)$ \\
\hline $\mathrm{O} 5-\mathrm{Ti} 3-\mathrm{O} 15$ & $89.68(7)$ & $\mathrm{O} 2-\mathrm{Mg} 4-\mathrm{O} 3$ & $79.20(7)$ \\
\hline $\mathrm{O} 5-\mathrm{Ti} 3-\mathrm{O} 16$ & $95.78(7)$ & $\mathrm{O} 2-\mathrm{Mg} 4-\mathrm{O} 4$ & $79.40(7)$ \\
\hline $\mathrm{O} 12-\mathrm{Ti3}-\mathrm{O} 14$ & $89.62(7)$ & $\mathrm{O} 2-\mathrm{Mg} 4-\mathrm{O} 17$ & $92.56(9)$ \\
\hline $\mathrm{O} 12-\mathrm{Ti} 3-\mathrm{O} 15$ & $164.80(7)$ & $\mathrm{O} 2-\mathrm{Mg} 4-\mathrm{O} 18$ & $94.71(8)$ \\
\hline $\mathrm{O} 12-\mathrm{Ti} 3-\mathrm{O} 16$ & $94.43(8)$ & $\mathrm{O} 2-\mathrm{Mg} 4-\mathrm{O} 19$ & $170.24(8)$ \\
\hline $\mathrm{O} 14-\mathrm{Ti3}-\mathrm{O} 15$ & $89.65(7)$ & $\mathrm{O} 3-\mathrm{Mg} 4-\mathrm{O} 4$ & $79.16(7)$ \\
\hline $\mathrm{O} 14-\mathrm{Ti3}-\mathrm{O} 16$ & $98.30(8)$ & $\mathrm{O} 3-\mathrm{Mg} 4-\mathrm{O} 17$ & $170.55(9)$ \\
\hline $\mathrm{O} 15-\mathrm{Ti3}-\mathrm{O} 16$ & $100.70(8)$ & $\mathrm{O} 3-\mathrm{Mg} 4-\mathrm{O} 18$ & $93.01(9)$ \\
\hline $\mathrm{O} 1-\mathrm{Mg} 1-\mathrm{O} 2$ & $84.92(6)$ & $\mathrm{O} 3-\mathrm{Mg} 4-\mathrm{O} 19$ & $93.70(8)$ \\
\hline $\mathrm{O} 1-\mathrm{Mg} 1-\mathrm{O} 3$ & $83.44(6)$ & $\mathrm{O} 4-\mathrm{Mg} 4-\mathrm{O} 17$ & $94.88(9)$ \\
\hline $\mathrm{O} 1-\mathrm{Mg} 1-\mathrm{O} 11$ & $74.24(6)$ & $\mathrm{O} 4-\mathrm{Mg} 4-\mathrm{O} 18$ & $170.91(9)$ \\
\hline $\mathrm{O} 1-\mathrm{Mg} 1-\mathrm{O} 14$ & $75.25(6)$ & $\mathrm{O} 4-\mathrm{Mg} 4-\mathrm{O} 19$ & $92.77(8)$ \\
\hline $\mathrm{O} 1-\mathrm{Mg} 1-\mathrm{Cl1}$ & 175.91(5) & $\mathrm{O} 17-\mathrm{Mg} 4-\mathrm{O} 18$ & $92.27(10)$ \\
\hline $\mathrm{O} 2-\mathrm{Mg} 1-\mathrm{O} 3$ & 79.74(7) & $\mathrm{O} 17-\mathrm{Mg} 4-\mathrm{O} 19$ & $93.91(9)$ \\
\hline $\mathrm{O} 2-\mathrm{Mg} 1-\mathrm{O} 11$ & $158.18(8)$ & $\mathrm{O} 18-\mathrm{Mg} 4-\mathrm{O} 19$ & 92.33(9) \\
\hline $\mathrm{O} 2-\mathrm{Mg} 1-\mathrm{O} 14$ & $92.84(7)$ & $\mathrm{Ti} 3-\mathrm{O} 1-\mathrm{Mg} 3$ & $88.27(6)$ \\
\hline $\mathrm{Ti} 1-\mathrm{O} 1-\mathrm{Ti} 2$ & $99.29(7)$ & $\mathrm{Mg} 1-\mathrm{O} 1-\mathrm{Mg} 2$ & $83.26(5)$ \\
\hline Ti1-O1-Ti3 & $98.47(6)$ & $\mathrm{Mg} 1-\mathrm{O} 1-\mathrm{Mg} 3$ & $82.27(5)$ \\
\hline Ti1-O5-Ti3 & $105.07(7)$ & $\mathrm{Mg} 1-\mathrm{O} 2-\mathrm{Mg} 3$ & $108.45(8)$ \\
\hline $\mathrm{Ti} 2-\mathrm{O} 1-\mathrm{Ti} 3$ & $99.04(7)$ & $\mathrm{Mg} 1-\mathrm{O} 2-\mathrm{Mg} 4$ & $98.64(8)$ \\
\hline $\mathrm{Ti} 1-\mathrm{O} 1-\mathrm{Mg} 1$ & $168.29(8)$ & $\mathrm{Mg} 2-\mathrm{O} 1-\mathrm{Mg} 3$ & $82.74(5)$ \\
\hline $\mathrm{Ti} 1-\mathrm{O} 1-\mathrm{Mg} 2$ & $88.84(6)$ & $\mathrm{Mg} 3-\mathrm{O} 2-\mathrm{Mg} 4$ & $97.81(7)$ \\
\hline $\mathrm{Ti} 1-\mathrm{O} 1-\mathrm{Mg} 3$ & $88.19(6)$ & $\mathrm{Mg} 2-\mathrm{O} 3-\mathrm{Mg} 1$ & $108.47(8)$ \\
\hline $\mathrm{Ti} 2-\mathrm{O} 1-\mathrm{Mg} 1$ & $89.19(6)$ & $\mathrm{Mg} 2-\mathrm{O} 3-\mathrm{Mg} 4$ & $98.94(8)$ \\
\hline $\mathrm{Ti} 2-\mathrm{O} 1-\mathrm{Mg} 2$ & $88.78(6)$ & $\mathrm{Mg} 1-\mathrm{O} 3-\mathrm{Mg} 4$ & $98.69(8)$ \\
\hline $\mathrm{Ti} 2-\mathrm{O} 1-\mathrm{Mg} 3$ & $168.59(8)$ & $\mathrm{Mg} 2-\mathrm{O} 4-\mathrm{Mg} 3$ & $108.51(8)$ \\
\hline $\mathrm{Ti} 3-\mathrm{O} 1-\mathrm{Mg} 1$ & $88.05(6)$ & $\mathrm{Mg} 2-\mathrm{O} 4-\mathrm{Mg} 4$ & $98.44(8)$ \\
\hline
\end{tabular}




\begin{tabular}{|c|c|c|c|}
\hline $\mathrm{Ti} 3-\mathrm{O} 1-\mathrm{Mg} 2$ & $168.24(8)$ & $\mathrm{Mg} 3-\mathrm{O} 4-\mathrm{Mg} 4$ & $98.74(7)$ \\
\hline \multicolumn{4}{|c|}{4} \\
\hline $\mathrm{Mg} 1-\mathrm{O} 1$ & $2.089(4)$ & $\mathrm{Ti} 1-\mathrm{O} 3$ & $1.996(3)$ \\
\hline $\mathrm{Mg} 1-\mathrm{O} 1^{\mathrm{i}}$ & $2.173(3)$ & Ti1-O6 & $1.920(3)$ \\
\hline $\mathrm{Mg} 1-\mathrm{O} 2^{\mathrm{i}}$ & $2.014(4)$ & $\mathrm{Ti1}-\mathrm{O} 7$ & $1.950(4)$ \\
\hline $\mathrm{Mg} 1-\mathrm{O} 3$ & $2.022(3)$ & Ti1-O8 & $1.792(3)$ \\
\hline $\mathrm{Mg} 1-\mathrm{O} 4$ & $2.105(3)$ & $\mathrm{O} 1-\mathrm{Ti} 1-\mathrm{O} 2$ & $80.68(13)$ \\
\hline $\mathrm{Mg} 1-\mathrm{O} 5$ & $2.069(4)$ & $\mathrm{O} 1-\mathrm{Ti} 1-\mathrm{O} 3$ & $77.76(13)$ \\
\hline $\mathrm{O} 1-\mathrm{Mg} 1-\mathrm{O} 1^{\mathrm{i}}$ & $85.23(13)$ & $\mathrm{O} 1-\mathrm{Ti1}-\mathrm{O} 6$ & $89.24(14)$ \\
\hline $\mathrm{O} 1-\mathrm{Mg} 1-\mathrm{O} 3$ & 77.07(13) & $\mathrm{O} 1-\mathrm{Ti} 1-\mathrm{O} 7$ & $85.53(14)$ \\
\hline $\mathrm{O} 1-\mathrm{Mg} 1-\mathrm{O} 4$ & $91.32(14)$ & $\mathrm{O} 1-\mathrm{Ti} 1-\mathrm{O} 8$ & $174.88(14)$ \\
\hline $\mathrm{O} 1-\mathrm{Mg} 1-\mathrm{O} 5$ & $175.31(14)$ & $\mathrm{O} 2-\mathrm{Ti} 1-\mathrm{O} 3$ & $85.22(13)$ \\
\hline $\mathrm{O} 1-\mathrm{Mg} 1-\mathrm{O} 3$ & $91.38(14)$ & $\mathrm{O} 2-\mathrm{Ti} 1-\mathrm{O} 6$ & $169.04(13)$ \\
\hline $\mathrm{O} 1-\mathrm{Mg} 1-\mathrm{O} 4$ & $173.39(14)$ & $\mathrm{O} 2-\mathrm{Ti} 1-\mathrm{O} 7$ & $85.80(14)$ \\
\hline $\mathrm{O} 1 \mathrm{i}-\mathrm{Mg} 1-\mathrm{O} 5$ & $90.08(14)$ & $\mathrm{O} 2-\mathrm{Ti} 1-\mathrm{O} 8$ & $94.95(14)$ \\
\hline $\mathrm{O} 2^{\mathrm{i}}-\mathrm{Mg} 1-\mathrm{O}^{\mathrm{i}}$ & 79.01(13) & $\mathrm{O} 3-\mathrm{Ti} 1-\mathrm{O} 6$ & $88.47(14)$ \\
\hline $\mathrm{O} 2 \mathrm{i}-\mathrm{Mg} 1-\mathrm{O} 1$ & $95.36(14)$ & $\mathrm{O} 3-\mathrm{Ti1}-\mathrm{O} 7$ & $162.11(13)$ \\
\hline $\mathrm{O} 2 \mathrm{i}-\mathrm{Mg} 1-\mathrm{O} 3$ & $168.32(15)$ & $\mathrm{O} 3-\mathrm{Ti} 1-\mathrm{O} 8$ & $99.29(15)$ \\
\hline $\mathrm{O} 2 \mathrm{i}-\mathrm{Mg} 1-\mathrm{O} 4$ & $106.98(15)$ & $\mathrm{O} 6-\mathrm{Ti} 1-\mathrm{O} 7$ & $97.75(15)$ \\
\hline $\mathrm{O} 2^{\mathrm{i}}-\mathrm{Mg} 1-\mathrm{O} 5$ & $83.47(14)$ & O6- $-\mathrm{Ti} 1-\mathrm{O} 8$ & $94.90(15)$ \\
\hline $\mathrm{O} 3-\mathrm{Mg} 1-\mathrm{O} 4$ & $82.34(14)$ & O7-Ti1-O8 & $96.88(15)$ \\
\hline $\mathrm{O} 3-\mathrm{Mg} 1-\mathrm{O} 5$ & $103.34(15)$ & $\mathrm{Ti} 1-\mathrm{O} 1-\mathrm{Mg} 1$ & $99.65(14)$ \\
\hline $\mathrm{O} 4-\mathrm{Mg} 1-\mathrm{O} 5$ & $93.37(15)$ & $\mathrm{Ti} 1-\mathrm{O} 1-\mathrm{Mg} 1^{\mathrm{i}}$ & $96.60(13)$ \\
\hline Ti1-O1 & $2.084(3)$ & $\mathrm{Mg} 1-\mathrm{O} 1-\mathrm{Mg} 1^{\mathrm{i}}$ & $94.77(13)$ \\
\hline $\mathrm{Ti} 1-\mathrm{O} 2$ & $2.035(3)$ & & \\
\hline \multicolumn{4}{|c|}{ 年 } \\
\hline $\mathrm{Ti1}-\mathrm{O} 1$ & $2.124(4)$ & $\mathrm{Mg} 1-\mathrm{O} 6$ & $2.047(5)$ \\
\hline $\mathrm{Ti} 1-\mathrm{O} 4$ & $1.958(4)$ & $\mathrm{Mg} 1-\mathrm{O} 8$ & $2.044(5)$ \\
\hline $\mathrm{Ti1}-\mathrm{O} 5$ & $1.964(4)$ & $\mathrm{Mg} 1-\mathrm{Cl} 1$ & $2.447(3)$ \\
\hline Ti1-O8 & $1.963(4)$ & $\mathrm{Mg} 2-\mathrm{O} 1$ & $2.339(4)$ \\
\hline $\mathrm{Ti1}-\mathrm{O} 10$ & $2.016(5)$ & $\mathrm{Mg} 2-\mathrm{O} 1^{\mathrm{i}}$ & $2.343(4)$ \\
\hline Ti1-O11 & $1.787(4)$ & $\mathrm{Mg} 2-\mathrm{O} 2$ & $2.010(4)$ \\
\hline $\mathrm{Ti} 2-\mathrm{O} 1$ & $2.123(4)$ & $\mathrm{Mg} 2-\mathrm{O} 3$ & $2.026(4)$ \\
\hline $\mathrm{Ti} 2-\mathrm{O} 6$ & $1.955(4)$ & $\mathrm{Mg} 2-\mathrm{O} 5$ & $2.034(4)$ \\
\hline $\mathrm{Ti} 2-\mathrm{O} 7$ & $1.964(4)$ & $\mathrm{Mg} 2-\mathrm{O}^{\mathrm{i}}$ & $2.022(4)$ \\
\hline Ti2-O9 & $1.962(4)$ & $\mathrm{Mg} 3-\mathrm{O} 1$ & $2.234(4)$ \\
\hline $\mathrm{Ti} 2-\mathrm{O} 10$ & $2.015(5)$ & $\mathrm{Mg} 3-\mathrm{O} 2$ & $2.053(4)$ \\
\hline $\mathrm{Ti} 2-\mathrm{O} 12$ & $1.785(5)$ & $\mathrm{Mg} 3-\mathrm{O} 3^{\mathrm{i}}$ & $2.061(4)$ \\
\hline $\mathrm{Mg} 1-\mathrm{O} 1$ & $2.281(4)$ & $\mathrm{Mg} 3-\mathrm{O} 4$ & $2.025(5)$ \\
\hline $\mathrm{Mg} 1-\mathrm{O} 2^{\mathrm{i}}$ & $2.045(4)$ & $\mathrm{Mg} 3-\mathrm{O} 9$ & $2.038(5)$ \\
\hline $\mathrm{Mg} 1-\mathrm{O} 3$ & $2.061(4)$ & $\mathrm{Mg} 3-\mathrm{O} 13$ & $2.073(5)$ \\
\hline $\mathrm{O} 1-\mathrm{Ti1}-\mathrm{O} 4$ & $83.63(16)$ & $\mathrm{O} 2-\mathrm{Mg} 2-\mathrm{O} 7^{\mathrm{i}}$ & $94.77(17)$ \\
\hline $\mathrm{O} 1-\mathrm{Ti} 1-\mathrm{O} 5$ & $88.17(15)$ & $\mathrm{O} 3-\mathrm{Mg} 2-\mathrm{O} 5$ & $95.29(17)$ \\
\hline $\mathrm{O} 1-\mathrm{Ti} 1-\mathrm{O} 8$ & $84.11(16)$ & $\mathrm{O} 3-\mathrm{Mg} 2-\mathrm{O} 7^{\mathrm{i}}$ & $95.52(18)$ \\
\hline $\mathrm{O} 1-\mathrm{Ti} 1-\mathrm{O} 10$ & $78.58(16)$ & $\mathrm{O} 5-\mathrm{Mg} 2-\mathrm{O} 7^{\mathrm{i}}$ & $103.73(18)$ \\
\hline $\mathrm{O} 1-\mathrm{Ti1}-\mathrm{O} 11$ & $173.5(2)$ & $\mathrm{O} 1 \mathrm{i}-\mathrm{Mg} 2-\mathrm{O} 3$ & $84.26(16)$ \\
\hline O4-Ti1-O5 & $88.84(17)$ & $\mathrm{O} 1-\mathrm{i} g 2-\mathrm{O} 5$ & $175.91(17)$ \\
\hline O4-Ti1-O8 & $167.69(18)$ & $\mathrm{O} 1^{\mathrm{i}}-\mathrm{Mg} 2-\mathrm{O}^{\mathrm{i}}$ & $80.36(16)$ \\
\hline $\mathrm{O} 4-\mathrm{Ti} 1-\mathrm{O} 10$ & $89.1(2)$ & $\mathrm{O} 2-\mathrm{Mg} 2-\mathrm{O} 3$ & $163.00(17)$ \\
\hline $\mathrm{O} 4-\mathrm{Ti1}-\mathrm{O} 11$ & $96.5(2)$ & $\mathrm{O} 2-\mathrm{Mg} 2-\mathrm{O} 5$ & $95.35(17)$ \\
\hline
\end{tabular}




\begin{tabular}{|c|c|c|c|}
\hline O5-Ti1-O8 & $89.72(19)$ & $\mathrm{O} 1-\mathrm{Mg} 3-\mathrm{O} 2$ & $86.27(15)$ \\
\hline $\mathrm{O} 5-\mathrm{Ti} 1-\mathrm{O} 10$ & $166.73(18)$ & $\mathrm{O} 1-\mathrm{Mg} 3-\mathrm{O} 3^{\mathrm{i}}$ & $86.30(15)$ \\
\hline $\mathrm{O} 5-\mathrm{Ti1}-\mathrm{O} 11$ & $98.4(2)$ & $\mathrm{O} 1-\mathrm{Mg} 3-\mathrm{O} 4$ & $79.35(16)$ \\
\hline $\mathrm{O} 8-\mathrm{Ti} 1-\mathrm{O} 10$ & $89.5(2)$ & $\mathrm{O} 1-\mathrm{Mg} 3-\mathrm{O} 9$ & $79.30(16)$ \\
\hline O8-Ti1-O11 & $95.8(2)$ & $\mathrm{O} 1-\mathrm{Mg} 3-\mathrm{O} 13$ & $179.6(2)$ \\
\hline $\mathrm{O} 10-\mathrm{Ti1}-\mathrm{O} 11$ & $94.9(2)$ & $\mathrm{O} 2-\mathrm{Mg} 3-\mathrm{O}^{\mathrm{i}}$ & $77.16(16)$ \\
\hline $\mathrm{O} 1-\mathrm{Ti} 2-\mathrm{O} 6$ & $84.73(16)$ & $\mathrm{O} 2-\mathrm{Mg} 3-\mathrm{O} 4$ & $93.95(17)$ \\
\hline $\mathrm{O} 1-\mathrm{Ti} 2-\mathrm{O} 7$ & $87.43(16)$ & $\mathrm{O} 2-\mathrm{Mg} 3-\mathrm{O} 13$ & $93.37(18)$ \\
\hline $\mathrm{O} 1-\mathrm{Ti} 2-\mathrm{O} 9$ & $83.76(16)$ & $\mathrm{O}^{\mathrm{i}}{ }^{-}-\mathrm{Mg} 3-\mathrm{O} 4$ & $163.63(19)$ \\
\hline $\mathrm{O} 1-\mathrm{Ti} 2-\mathrm{O} 10$ & $78.62(16)$ & $\mathrm{O}^{\mathrm{i}}-\mathrm{Mg} 3-\mathrm{O} 9$ & $92.68(19)$ \\
\hline $\mathrm{O} 1-\mathrm{Ti} 2-\mathrm{O} 12$ & $174.0(2)$ & $\mathrm{O} 3^{\mathrm{i}}-\mathrm{Mg} 3-\mathrm{O} 13$ & $93.79(19)$ \\
\hline $\mathrm{O} 6-\mathrm{Ti} 2-\mathrm{O} 7$ & $88.78(18)$ & $\mathrm{O} 4-\mathrm{Mg} 3-\mathrm{O} 9$ & $92.4(2)$ \\
\hline O6-Ti2-O9 & $168.26(18)$ & $\mathrm{O} 4-\mathrm{Mg} 3-\mathrm{O} 13$ & $100.5(2)$ \\
\hline $\mathrm{O} 6-\mathrm{Ti} 2-\mathrm{O} 10$ & $89.9(2)$ & $\mathrm{O} 9-\mathrm{Mg} 3-\mathrm{O} 2$ & $162.91(19)$ \\
\hline $\mathrm{O} 6-\mathrm{Ti} 2-\mathrm{O} 12$ & $95.2(2)$ & $\mathrm{O} 9-\mathrm{Mg} 3-\mathrm{O} 13$ & 101.07(19) \\
\hline O7-Ti2-O9 & $88.41(19)$ & $\mathrm{Ti} 1-\mathrm{O} 1-\mathrm{Ti} 2$ & $97.74(15)$ \\
\hline $\mathrm{O} 7-\mathrm{Ti} 2-\mathrm{O} 10$ & $166.04(17)$ & $\mathrm{Ti} 1-\mathrm{O} 1-\mathrm{Mg} 1$ & $92.37(14)$ \\
\hline $\mathrm{O} 7-\mathrm{Ti} 2-\mathrm{O} 12$ & $98.6(2)$ & $\mathrm{Ti1}-\mathrm{O} 1-\mathrm{Mg} 2$ & $88.48(14)$ \\
\hline $\mathrm{O} 9-\mathrm{Ti} 2-\mathrm{O} 10$ & $90.1(2)$ & $\mathrm{Ti} 1-\mathrm{O} 1-\mathrm{Mg} 2^{\mathrm{i}}$ & $173.44(19)$ \\
\hline $\mathrm{O} 9-\mathrm{Ti} 2-\mathrm{O} 12$ & $96.4(2)$ & Ti1-O1-Mg3 & $92.47(15)$ \\
\hline $\mathrm{O} 10-\mathrm{Ti2}-\mathrm{O} 12$ & $95.4(2)$ & $\mathrm{Ti} 1-\mathrm{O} 4-\mathrm{Mg} 3$ & $104.44(19)$ \\
\hline $\mathrm{O} 1-\mathrm{Mg} 1-\mathrm{O} 2^{\mathrm{i}}$ & $85.0(2)$ & $\mathrm{Ti} 1-\mathrm{O} 5-\mathrm{Mg} 2$ & $102.46(18)$ \\
\hline $\mathrm{O} 1-\mathrm{Mg} 1-\mathrm{O} 3$ & $84.86(15)$ & $\mathrm{Ti} 1-\mathrm{O} 8-\mathrm{Mg} 1$ & $105.04(19)$ \\
\hline $\mathrm{O} 1-\mathrm{Mg} 1-\mathrm{O} 6$ & $78.72(17)$ & $\mathrm{Ti} 1-\mathrm{O} 10-\mathrm{Ti} 2$ & $105.06(19)$ \\
\hline $\mathrm{O} 1-\mathrm{Mg} 1-\mathrm{O} 8$ & $78.41(16)$ & $\mathrm{Ti} 2-\mathrm{O} 1-\mathrm{Mg} 1$ & $91.87(15)$ \\
\hline $\mathrm{O} 1-\mathrm{Mg} 1-\mathrm{Cl} 1$ & $177.19(14)$ & $\mathrm{Ti} 2-\mathrm{O} 1-\mathrm{Mg} 2$ & $173.77(19)$ \\
\hline $\mathrm{O} 2 \mathrm{i}-\mathrm{Mg} 1-\mathrm{O} 3$ & $77.35(16)$ & $\mathrm{Ti} 2-\mathrm{O} 1-\mathrm{Mg} 2^{\mathrm{i}}$ & $88.80(14)$ \\
\hline $\mathrm{O} 22^{\mathrm{i}}-\mathrm{Mg} 1-\mathrm{O} 6$ & $93.56(17)$ & $\mathrm{Ti} 2-\mathrm{O} 1-\mathrm{Mg} 3$ & $92.77(15)$ \\
\hline $\mathrm{O} 2 \mathrm{i}-\mathrm{Mg} 1-\mathrm{O} 8$ & $161.34(19)$ & $\mathrm{Ti} 2-\mathrm{O} 7-\mathrm{Mg} 2^{\mathrm{i}}$ & $103.41(19)$ \\
\hline $\mathrm{O} 2^{\mathrm{i}}-\mathrm{Mg} 1-\mathrm{Cl}$ & $96.66(14)$ & $\mathrm{Mg} 1-\mathrm{O} 1-\mathrm{Mg} 2$ & $87.67(14)$ \\
\hline $\mathrm{O} 3-\mathrm{Mg} 1-\mathrm{O} 6$ & $161.91(18)$ & $\mathrm{Mg} 1-\mathrm{O} 1-\mathrm{Mg}^{\mathrm{i}}$ & $87.02(14)$ \\
\hline $\mathrm{O} 3-\mathrm{Mg} 1-\mathrm{O} 8$ & $92.59(18)$ & $\mathrm{Mg} 1-\mathrm{O} 1-\mathrm{Mg} 3$ & $172.79(19)$ \\
\hline $\mathrm{O} 3-\mathrm{Mg} 1-\mathrm{Cl} 1$ & $97.72(14)$ & $\mathrm{Mg} 1-\mathrm{O} 3-\mathrm{Mg} 2$ & $103.08(18)$ \\
\hline $\mathrm{O} 6-\mathrm{Mg} 1-\mathrm{O} 8$ & $91.5(2)$ & $\mathrm{Mg} 1-\mathrm{O} 3-\mathrm{Mg}^{\mathrm{i}}$ & $100.50(18)$ \\
\hline $\mathrm{O} 6-\mathrm{Mg} 1-\mathrm{Cl} 1$ & $98.87(15)$ & $\mathrm{Mg} 2-\mathrm{O} 1-\mathrm{Mg} 3$ & $87.13(14)$ \\
\hline $\mathrm{O} 8-\mathrm{Mg} 1-\mathrm{Cl} 1$ & $100.28(15)$ & $\mathrm{Mg} 2-\mathrm{O} 2-\mathrm{Mg}^{\mathrm{i}}$ & $103.49(18)$ \\
\hline $\mathrm{O} 1-\mathrm{Mg} 2-\mathrm{O}^{\mathrm{i}}$ & $95.03(13)$ & $\mathrm{Mg} 1-\mathrm{O} 2-\mathrm{Mg} 3$ & $101.32(17)$ \\
\hline $\mathrm{O} 1-\mathrm{Mg} 2-\mathrm{O} 2$ & $84.50(15)$ & $\mathrm{Mg} 2-\mathrm{O} 1-\mathrm{Mg} 2^{\mathrm{i}}$ & $84.97(13)$ \\
\hline $\mathrm{O} 1-\mathrm{Mg} 2-\mathrm{O} 3$ & $84.15(16)$ & $\mathrm{Mg} 2-\mathrm{O} 2-\mathrm{Mg} 3$ & $101.76(17)$ \\
\hline $\mathrm{O} 1-\mathrm{Mg} 2-\mathrm{O} 5$ & $80.89(16)$ & $\mathrm{Mg} 2-\mathrm{O} 3-\mathrm{Mg} 3^{\mathrm{i}}$ & 101.61(18) \\
\hline $\mathrm{O} 1-\mathrm{Mg} 2-\mathrm{O}^{\mathrm{i}}$ & $175.38(18)$ & $\mathrm{Mg} 3-\mathrm{O} 1-\mathrm{Mg} 2^{\mathrm{i}}$ & $87.56(14)$ \\
\hline $\mathrm{O} 1 \mathrm{i}-\mathrm{Mg} 2-\mathrm{O} 2$ & $84.18(15)$ & & \\
\hline
\end{tabular}

